\title{
Homotopy Analysis Method for the First Order Fuzzy Volterra-Fredholm Integro-Differential Equations
}

\author{
Ahmed A. Hamoud ${ }^{1}$ and Kirtiwant P. Ghadle ${ }^{2}$ \\ ${ }^{1,2}$ Department of Mathematics, Dr. Babasaheb Ambedkar Marathwada University, Aurangabad-431004 (M.S.) India \\ ${ }^{1}$ Department of Mathematics, Taiz University, Taiz, Yemen
}

\section{Article Info}

\section{Article history:}

Received, April 28, 2018

Revised, Jul 22, 2018

Accepted, Aug 4, 2018

\section{Keyword:}

Homotopy analysis method

Fuzzy Volterra-Fredholm

integro-differential equation

Existence and uniqueness

results.

\section{Corresponding Author:}

Ahmed A. Hamoud

Department of Mathematics,

Taiz University,

Taiz, Yemen.

Email: drahmed985@yahoo.com

\begin{abstract}
A fuzzy Volterra-Fredholm integro-differential equation (FVFIDE) in a parametric case is converted to its related crisp case. We use homotopy analysis method to find the approximate solution of this system and hence obtain an approximation for the fuzzy solution of the FVFIDE. This paper discusses existence and uniqueness results and convergence of the proposed method.
\end{abstract}

Copyright (c) 2018 Institute of Advanced Engineering and Science. All rights reserved.

\section{INTRODUCTION}

In recent years, the topics of fuzzy integral equations which attracted increasing interest, in particular in relation to fuzzy control, have been rapidly developed. The concept of fuzzy numbers and arithmetic operations firstly introduced by Zadeh [1], and then by Dubois and Prade. Also, in [2] have introduced the concept of integration of fuzzy functions. The fuzzy mapping function was introduced by Cheng and Zadeh [1]. Moreover, Dubois and Prade [3] presented an elementary fuzzy calculus based on the extension principle. The fuzzy integro-differential equations are a natural way to model uncertainty of dynamical systems. Kaleva [4] chose to define the integral of the fuzzy function, using the Lebesgue-type concept for integration. Recently, Hence various other methods for solving them such as using homotopy perturbation method [5], expansion method [6], Laplace transformation method [7], homotopy analysis method [8], differential transform method [9], fixed point theorems [10], variational iteration method [11]. Also, some mathematicians have studied fuzzy integral and integro-differential equation by numerical techniques [12]$[21][23,26]$. As we know the fuzzy integral and integro-differential equations are one of the important parts of the fuzzy analysis theory that play a main role in the numerical analysis.

In this work, we will examine HAM to approximate the solution of the fuzzy Volterra-Fredholm integrodifferential equation of the second kind. The structure of this paper is organized as follows: In Section 2, we state some known notations and definitions and also some theorems which are used throughout this paper. In Section 3, the fuzzy Volterra-Fredholm integro-differential equation of the second kind is briefly presented. In Section 4, we convert a fuzzy Volterra-Fredholm integro-differential equation of the second kind to the system of Volterra-Fredholm integro-differential equation of the second kind in a crisp case and approximate with HAM. In Section 5, the existence and uniqueness results and convergence of the proposed method is proved. In Section 6, the analytical example is presented illustrate the accuracy of this method. Finally, we will give a report on our paper and a brief conclusion in Section 7. 


\section{PRELIMINARIES}

The concept of fuzzy numbers is generalized of classical real numbers and we can say that a fuzzy number is a fuzzy subset of the real line which has some additional properties. The concept of fuzzy number is vital for fuzzy analysis, fuzzy integral equations and fuzzy differential equations, and a very helpful tool in different applications of fuzzy sets. Basic definition of fuzzy numbers is given in [1, 2, 3, 27].

Definition 2..1 [2] Let us denote by $\mathbb{R}_{\mathcal{F}}$ the class of fuzzy subsets of the real axis $u: \mathbb{R} \longrightarrow I=[0,1]$, satisfying the following properties:

- $u$ is upper semi-continuous function,

- $u$ is fuzzy convex,i.e, $u(\lambda x+(1-\lambda) y) \geq \min \{u(x), u(y)\}$ for all $x, y \in \mathbb{R}, \lambda \in[0,1]$,

- $u$ is normal, i.e, $\exists x_{0} \in \mathbb{R}$ for which $u\left(x_{0}\right)=1$,

- $\sup u=\{x \in \mathbb{R} \mid u(x)>0\}$ is the support of the $u$, and its closure cl( $\sup u)$ is compact.

Let $\mathbb{E}$ be the set of all fuzzy numbers on $\mathbb{R}_{\mathcal{F}}$. The $(\alpha-$ cut $) \alpha$-level set of a fuzzy number $u \in \mathbb{E}, 0 \leq \alpha \leq 1$, denoted by $[u]_{\alpha}$, is defined as

$$
[u]_{\alpha}= \begin{cases}\{x \in \mathbb{R}: u(x) \geq \alpha\}, & 0<\alpha \leq 1, \\ c l(\sup u), & \alpha=0 .\end{cases}
$$

where $c l(\sup u=x \in \mathbb{R} \mid u(x)>0)$ denotes the closure of the support of $u$. It is clear that the $\alpha$-level set of a fuzzy number is a closed and bounded interval $[\underline{u}(\alpha), \bar{u}(\alpha)]$, where $\underline{u}(\alpha)$ denotes the left-hand end point of $[u]_{\alpha}$, and $\bar{u}(\alpha)$ denotes the right-hand end point of $[u]_{\alpha}$. Since each $u \in \mathbb{R}$ can be regarded as a fuzzy number $\tilde{u}$ defined by:

$$
\tilde{u}(t)= \begin{cases}1, & t=u \\ 0, & t \neq u\end{cases}
$$

An equivalent parametric definition is also given in [1] as:

Definition 2..2 [2] A fuzzy number $\tilde{u}$ in parametric form is a pair $(\underline{u}, \bar{u})$ of functions $\underline{u}(\alpha), \bar{u}(\alpha), 0 \leq \alpha \leq 1$, which satisfy the following requirements:

- $\underline{u}(\alpha)$ is a bounded non-decreasing left continuous function in $(0,1]$, and right continuous at 0 ,

- $\bar{u}(\alpha)$ is a bounded non-increasing left continuous function in $(0,1]$, and right continuous at 0 ,

- $\underline{u}(\alpha) \leq \bar{u}(\alpha), 0 \leq \alpha \leq 1$.

A crisp number $\alpha$ is simply represented by $\underline{u}(\alpha)=\bar{u}(\alpha)=\alpha, 0 \leq \alpha \leq 1$. We recall that for $a<b<c$ which $a, b, c \in \mathbb{R}$, the triangular fuzzy number $u=(a, b, c)$ determined by $a, b, c$ are given such that $\underline{u}(\alpha)=a+(b-a) \alpha$ and $\bar{u}(\alpha)=c-(c-b) \alpha$ are the end points of the $\alpha$-level sets, for all $\alpha \in[0,1]$.

The Hausdorff distance between fuzzy numbers given by

$$
\begin{gathered}
D: \mathbb{R}_{\mathcal{F}} \times \mathbb{R}_{\mathcal{F}} \rightarrow \mathbb{R}_{+} \cup\{0\} . \\
D(u, v)=\sup _{\alpha \in[0,1]} \max \{|\underline{u}(\alpha)-\underline{v}(\alpha)|,|\bar{u}(\alpha)-\bar{v}(\alpha)|\}
\end{gathered}
$$

wehre $u=(\underline{u}(\alpha), \bar{u}(\alpha)), v=(\underline{v}(\alpha), \bar{v}(\alpha)) \subset \mathbb{R}$ is utilized in [1]. Then, it is easy to see that $d$ is a metric in $\mathbb{E}$ and has the following properties:

- $D(u+\rho, v+\rho)=D(u, v), \quad \forall u, v, \rho \in \mathbb{E}$,

- $D(k u, k v)=|k| D(u, v), \quad \forall k \in \mathbb{R} ; u, v \in \mathbb{E}$,

- $D(\omega+v, \rho+e) \leq D(\omega, \rho)+d(v, e), \quad \forall \omega, v, \rho, e \in \mathbb{E}$,

- $(D, \mathbb{E})$ is a complete metric space. 
Definition 2..3 The function $f:[a, b] \longrightarrow \mathbb{R}_{\mathcal{F}}$ is called a Lipschitz function if there exists a real constant $L \geq 0$ such that, for all $x, t \in[a, b]$

$$
D(f(x), f(t)) \leq L|x-t|
$$

We refer to $L$ as the Lipschitz constant of the function $f$.

Remark 2..1 Let $u(\alpha)=(\underline{u}(\alpha), \bar{u}(\alpha))$, be a fuzzy number, we take

$$
u^{c}(\alpha)=\frac{\underline{u}(\alpha)+\bar{u}(\alpha)}{2}, \quad u^{d}(\alpha)=\frac{\bar{u}(\alpha)-\underline{u}(\alpha)}{2} .
$$

It is clear that $u^{d}(\alpha) \geq 0$ and $\underline{u}(\alpha)=u^{c}(\alpha)-u^{d}(\alpha)$ and $\bar{u}(\alpha)=u^{c}(\alpha)+u^{d}(\alpha)$, also a fuzzy number $u \in \mathbb{E}$ is said symmetric if $u^{c}(\alpha)$ is independent of $\alpha$ for all $0 \leq \alpha \leq 1$.

Definition 2..4 Let $f: \mathbb{R} \rightarrow \mathbb{E}$ be a fuzzy valued function. If for arbitrary fixed $t_{0} \in \mathbb{R}$ and $\forall \epsilon>0, \exists \delta>0$ such that $\left|t-t_{0}\right|<\delta \Longrightarrow\left|f(t)-f\left(t_{0}\right)\right|<\epsilon, f$ is said to be continuous.

Theorem 2..2 Let $f(x)$ be a fuzzy-valued function on $[a, \infty)$ and it is represented by $(f(x, \alpha), \bar{f}(x, \alpha))$. For any fixed $t \in[0,1]$ assume $\underline{f}(x, \alpha)$ and $\bar{f}(x, \alpha)$ are Riemann-integrable on $[a, b]$ for every $b \geq a$, and assume there are two positive $\underline{M}(\alpha)$ and $\bar{M}(\alpha)$ such that $\int_{a}^{b}|\underline{f}(x, \alpha)| d x \leq \underline{M}(\alpha)$ and $\int_{a}^{b}|\bar{f}(x, \alpha)| d x \leq \bar{M}(\alpha)$ for every $b \geq a$. Then $f(x)$ is improper fuzzy Riemann-integrable on $[a, \infty)$ and the improper fuzzy Riemann-integral is a fuzzy number. Furthermore, we have:

$$
\int_{a}^{\infty} f(x) d x=\left(\int_{a}^{\infty} \underline{f}(x, \alpha) d x, \int_{a}^{\infty} \bar{f}(x, \alpha) d x\right)
$$

Proposition 2..3 [25]. If each of $f(x)$ and $g(x)$ is fuzzy-valued function and fuzzy Riemman integrable on $\Omega=[a, \infty)$ then $f(x)+g(x)$ is fuzzy Riemman integrable on $\Omega$. Moreover, we have:

$$
\int_{\Omega}(f(x)+g(x)) d x=\int_{\Omega} f(x) d x+\int_{\Omega} g(x) d x
$$

Definition 2..5 [25] The integral of a fuzzy function was define by using the Riemann integral concept. Let $f:[a, b] \rightarrow$ $\mathbb{E}$, for each partition $P=t_{0}, t_{1}, \ldots, t_{n}$ of $[a, b]$ and for arbitrary $\xi_{i} \in\left[t_{i-1}, t_{i}\right], 1 \leq i \leq n$, suppose

$$
\begin{gathered}
R_{p}=\sum_{i=1}^{n} f\left(\xi_{i}\right)\left(t_{i}-t_{i-1}\right) \\
\Delta:=\max \left|t_{i}-t_{i-1}\right|, \quad 1 \leq i \leq n .
\end{gathered}
$$

The definite integral of $f(t)$ over $[a, b]$ is

$$
\int_{a}^{b} f(t) d t=\lim _{\Delta \rightarrow 0} R_{p}
$$

Provided that this limit exists in the metric d. If the fuzzy function $f(t)$ is continuous in the metric d, its definite integral exists, and also

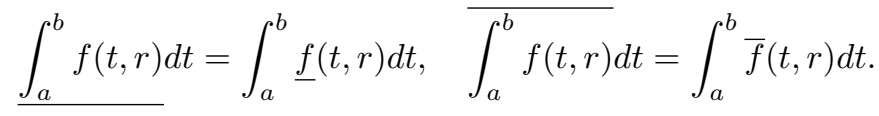

More details about the properties of the fuzzy integral are given in [2, 26, 25].

Theorem 2..4 [28] (Banach contraction principle). Let $(X, d)$ be a complete metric space, then each contraction mapping $\mathcal{T}: X \longrightarrow X$ has a unique fixed point $x$ of $\mathcal{T}$ in $X$ i.e. $\mathcal{T} x=x$.

Theorem 2..5 [24] (Schauder's fixed point theorem). Let X be a Banach space and let A a convex, closed subset of $X$. If $T: A \longrightarrow A$ be the map such that the set $\{T u: u \in A\}$ is relatively compact in $X$ (or $T$ is continuous and completely continouous). Then $T$ has at least one fixed point $u^{*} \in A: T u^{*}=u^{*}$. 


\section{FUZZY VOLTERRA-FREDHOLM INTEGRO-DIFFERENTIAL EQUATION}

In this section we consider the fuzzy Volterra-Fredholm integro-differential equation

$$
\tilde{u}^{\prime}(x)=\tilde{f}(x)+\lambda \int_{a}^{x} k_{1}(x, t) F_{1}(\tilde{u}(t)) d t+\mu \int_{a}^{\beta} k_{2}(x, t) F_{2}(\tilde{u}(t)) d t,
$$

with initial condition

$$
\tilde{u}_{0}(0)=\tilde{u}(0)
$$

where $\lambda, \mu \in \mathbb{R}, f(x), k_{1}, k_{2}$ and $F_{1}(\tilde{u}(t))$ are analytical functions $k_{1}, k_{2}: \mathbb{C}\left([0, \beta]^{2}\right) \longrightarrow \mathbb{R}^{+}$, that have suitable derivatives on an interval $0 \leq t \leq x \leq \beta$ and $\tilde{u}(x)$ is unknown function. The solution is expressed in the form:

$$
\tilde{u}(x)=\sum_{i=0}^{\infty} \tilde{u}_{i}(x)
$$

Let

$$
\tilde{u}(x, t)=(\underline{u}(x, t), \bar{u}(x, t)), \quad \tilde{f}(x, t)=(\underline{f}(x, t), \bar{f}(x, t)) .
$$

and

$$
\tilde{u}^{\prime}(x, t)=\left(\underline{u}^{\prime}(x, t), \bar{u}^{\prime}(x, t)\right), \quad \tilde{f}^{\prime}(x, t)=\left(\underline{f}^{\prime}(x, t), \bar{f}^{\prime}(x, t)\right) .
$$

Therefore, the related fuzzy integro-differential equation (1) can be written as follows

$$
\begin{aligned}
& \underline{u}^{\prime}(x, t)=\underline{f}(x, t)+\lambda \int_{a}^{x} k_{1}(x, s) F_{1}(\underline{u}(s, t)) d s+\mu \int_{a}^{\beta} k_{2}(x, s) F_{2}(\underline{u}(s, t)) d s \\
& \bar{u}^{\prime}(x, t)=\bar{f}(x, t)+\lambda \int_{a}^{x} k_{1}(x, s) F_{1}(\bar{u}(s, t)) d s+\mu \int_{a}^{\beta} k_{2}(x, s) F_{2}(\bar{u}(s, t)) d s
\end{aligned}
$$

Similar to Remark 2.1, let

$$
u^{c}(x, t)=\frac{\underline{u}(x, t)+\bar{u}(x, t)}{2}, \quad u^{d}(x, t)=\frac{\bar{u}(x, t)-\underline{u}(x, t)}{2} .
$$

and

$$
f^{c}(x, t)=\frac{f(x, t)+\bar{f}(x, t)}{2}, \quad f^{d}(x, t)=\frac{\bar{f}(x, t)-\underline{f}(x, t)}{2} .
$$

then (4) and (5) can be written as

$$
\begin{aligned}
& u^{\prime c}(x, t)=f^{c}(x, t)+\lambda \int_{a}^{x} k_{1}(x, s) F_{1}\left(u^{c}(s, t)\right) d s+\mu \int_{a}^{\beta} k_{2}(x, s) F_{2}\left(u^{c}(s, t)\right) d s \\
& u^{\prime d}(x, t)=f^{d}(x, t)+\lambda \int_{a}^{x} k_{1}(x, s) F_{1}\left(u^{d}(s, t)\right) d s+\mu \int_{a}^{\beta} k_{2}(x, s) F_{2}\left(u^{d}(s, t)\right) d s
\end{aligned}
$$

and

$$
u^{c}(0, t)=\frac{u}{(0, t)+\bar{u}(0, t)}, \quad u^{d}(0, t)=\frac{\bar{u}(0, t)-\underline{u}(0, t)}{2} .
$$

\section{HOMOTOPY ANALYSIS METHOD (HAM)}

In this section, we shall describe the solution approaches based on HAM for fuzzy Volterra-Fredholm integrodifferential equations. For this, we consider the first equation of (8) namely we apply HAM for finding $u^{c}(x, t)$, and second equation approach is similar to the first one [22]. Consider

$$
u^{\prime c}(x, t)=f^{c}(x, t)+\lambda \int_{a}^{x} k_{1}(x, s) F_{1}\left(u^{c}(s, t)\right) d s+\mu \int_{a}^{\beta} k_{2}(x, s) F_{2}\left(u^{c}(s, t)\right) d s
$$


with initial condition $u^{c}(0, t)$. We first construct the zero-order deformation equation

$$
(1-q) \Psi\left[\varphi(x, t ; q)-u_{0}^{c}(x, t)\right]=q \hbar N[\varphi(x, t ; q)]
$$

subject to the initial condition

$$
\varphi(0, t ; q)=u_{0}^{c}(x, t)=u^{c}(0, t),
$$

where $q \in[0,1]$ is the embedding parameter and $\hbar \neq 0$ is an auxiliary parameter and

$$
\Psi[\varphi(x, t ; q)]=\frac{\partial[\varphi(x, t ; q)]}{\partial x},
$$

with the property

$$
\Psi[C]=0,
$$

where $C$ is integral constant. Also from (11), we can define

$$
\begin{aligned}
N[\varphi(x, t ; q)]= & \frac{\partial[\varphi(x, t ; q)]}{\partial x}-f^{c}(x, t)-\lambda \int_{a}^{x} k_{1}(x, s) F_{1}(\varphi(s, t ; q)) d s \\
& -\mu \int_{a}^{\beta} k_{2}(x, s) F_{2}(\varphi(s, t ; q)) d s,
\end{aligned}
$$

When parameter of $q$ increases from 0 to 1 , then homotopy solution $\varphi(x, t ; q)$ varies from $u_{0}^{c}(x, t)$ to solution $u^{c}(t, r)$ of the original equation (11). Using the parameter $q, \varphi(x, t ; q)$ can be expanded in Taylor series as follows

$$
\varphi(x, t ; q)=u_{0}^{c}(x, t)+\sum_{m=0}^{\infty} u_{m}^{c}(x, t) q^{m},
$$

where

$$
u_{m}^{c}(x, t)=\left.\frac{1}{m !} \frac{\partial^{m}[\varphi(x, t ; q)]}{\partial^{m} q}\right|_{q=0}
$$

Assuming that auxiliary parameter $\hbar$ is properly selected so that the above series is convergent when $q=1$, then the solution $u^{c}(x, t)$ can be given by

$$
u^{c}(x, t)=u_{0}^{c}(x, t)+\sum_{m=0}^{\infty} u_{m}^{c}(x, t) .
$$

Differentiating (12) and initial condition (13) m-times with respect to $q$, then setting $q=0$, and finally dividing them by $m$ !, we gain the $m^{t h}$-order deformation equation

$$
\Psi\left[u_{m}^{c}(x, t)-\chi_{m} u_{m-1}^{c}(x, t)\right]=\hbar \Re_{m} \overrightarrow{\left(u_{m-1}^{c}\right)},
$$

subject to the following initial conditions,

$$
u_{m}^{c}(0, t)=0
$$

where

$$
\begin{aligned}
\Re_{m} \overrightarrow{\left(u_{m-1}^{c}\right)}= & \left.\frac{1}{(m-1) !} \frac{\partial^{m-1} N[\varphi(x, t ; q)]}{\partial^{m-1} q}\right|_{q=0} \\
= & \frac{\partial u_{m-1}^{c}(x, t)}{\partial x}-\left(1-\chi_{m}\right) f^{c}(x, t) \\
& -\lambda \int_{a}^{x} k_{1}(x, s) F_{1}\left(u_{m-1}^{c}(s, t)\right) d s-\mu \int_{a}^{\beta} k_{2}(x, s) F_{2}\left(u_{m-1}^{c}(s, t)\right) d s,
\end{aligned}
$$

and

$$
\chi_{m}= \begin{cases}0 & m \leq 1 \\ 1 & m>1 .\end{cases}
$$

Note that the high-order deformation Eq.(17) is governing the linear operator $L$, and the term $\Re_{m}\left(\overrightarrow{u_{m-1}^{c}}\right)$ can be expressed simply by Eq.(19) for any nonlinear operator $N$. Similarly, we can apply HAM for finding $u^{d}(x, t)$. 


\section{MAIN RESULTS}

A function $\tilde{u}$ is a solution of the initial value problem (1)-(2) if and only if it is continuous and satisfies the integral equation

$$
\tilde{u}(x)=\tilde{u}_{0}+\int_{a}^{x} \tilde{f}(t) d t+\int_{a}^{x} \int_{a}^{t} \lambda k_{1}(t, s) F_{1}(\tilde{u}(s)) d s d t+\int_{a}^{x} \int_{a}^{\beta} \mu k_{2}(t, s) F_{2}(\tilde{u}(s)) d s d t,
$$

By changing the order of the integration, we have

$$
\begin{aligned}
& \tilde{u}(x)=\tilde{u}_{0}+\int_{a}^{x} \tilde{f}(t) d t+\int_{a}^{x} \int_{s}^{x} \lambda k_{1}(t, s) F_{1}(\tilde{u}(s)) d t d s+\int_{a}^{x} \int_{a}^{\beta} \mu k_{2}(t, s) F_{2}(\tilde{u}(s)) d s d t, \\
& \tilde{u}(x)=\tilde{u}_{0}+\int_{a}^{x} \tilde{f}(t) d t+\int_{a}^{x} \int_{s}^{x} \lambda k_{1}(t, s) F_{1}(\tilde{u}(s)) d t d s+\int_{a}^{\beta} \int_{a}^{x} \mu k_{2}(t, s) F_{2}(\tilde{u}(s)) d t d s,
\end{aligned}
$$

Since the function $k_{1}$ and $k_{1}$ are with no sign changes by assumption, we have

$$
\begin{aligned}
& \int_{s}^{x} \lambda k_{1}(t, s) F_{1}(\tilde{u}(s)) d t=\int_{s}^{x} \lambda k_{1}(t, s) d t \cdot F_{1}(\tilde{u}(s)) \\
& \int_{a}^{x} \mu k_{2}(t, s) F_{2}(\tilde{u}(s)) d t=\int_{a}^{x} \mu k_{2}(t, s) d t \cdot F_{2}(\tilde{u}(s))
\end{aligned}
$$

thus

$$
\tilde{u}(x)=\tilde{g}(x)+\lambda \int_{a}^{x} K_{1}(x, s) F_{1}(\tilde{u}(s)) d s+\mu \int_{a}^{\beta} K_{2}(x, s) F_{2}(\tilde{u}(s)) d s
$$

where

$$
\tilde{g}(x)=\tilde{u}_{0}+\int_{a}^{x} \tilde{f}(t) d t, \quad K_{1}(x, s)=\int_{s}^{x} k_{1}(t, s) d t, \quad K_{2}(x, s)=\int_{a}^{x} k_{2}(t, s) d t .
$$

Lemma 5..1 Let $k_{1}(t, s)$ be continuous in $(t, s)$ and Lipschitz with respect to $s$. Then $K_{1}(x, s)$ is Lipschitz with respect to $s$.

Proof. Let $0 \leq s_{1} \leq s_{2} \leq x$. Then

$$
\begin{aligned}
\left|K_{1}\left(x, s_{1}\right)-K_{1}\left(x, s_{2}\right)\right| & =\left|\int_{s_{1}}^{x} k_{1}\left(t, s_{1}\right) d t-\int_{s_{2}}^{x} k_{1}\left(t, s_{2}\right) d t\right| \\
& =\left|\int_{s_{1}}^{s_{2}} k_{1}\left(t, s_{1}\right) d t+\int_{s_{2}}^{x} k_{1}\left(t, s_{1}\right) d t-\int_{s_{2}}^{x} k_{1}\left(t, s_{2}\right) d t\right| \\
& \leq \int_{s_{1}}^{s_{2}}\left|k_{1}\left(t, s_{1}\right)\right| d t+\int_{s_{2}}^{x}\left|k_{1}\left(t, s_{1}\right)-k_{1}\left(t, s_{2}\right)\right| d t \\
& \leq M\left|s_{1}-s_{2}\right|+L\left|s_{1}-s_{2}\right|\left(x-s_{2}\right) \\
& \leq(M+L(\beta-a))\left|s_{1}-s_{2}\right|,
\end{aligned}
$$

where $M=\max _{(x, s) \in G}\left|k_{1}(x, s)\right|, G:=\{(x, t) \mid x \in J, t \in[a, x]\} \subset J \times J$, and $L$ is the Lipschitz constant of $k_{1}$ and thus $K_{1}$ satisfies in Lipschitz condition. Similarly, we can proof the procedure of $K_{2}(x, s)$ is Lipschitz with respect to $s$. Before starting and proving the main results, we introduce the following hypotheses:

(A1) There exist two constants $M_{1}, M_{2}>0$ such that, for any $u_{1}, u_{2} \in C(J, \mathbb{R})$

$$
D_{\varepsilon}\left(u_{1}^{c}, u_{2}^{c}\right):=\sup _{x \in J} e^{-\varepsilon M_{1} x} D\left(u_{1}^{c}(s), u_{2}^{c}(s)\right), \varepsilon \geq 1
$$

as a metric on $X$.

(A2) There exist two functions $K_{1}, K_{2} \in C\left(D, \mathbb{R}^{+}\right)$, the set of all positive function continuous on $D=\{(x, t) \in$ $\mathbb{R} \times \mathbb{R}: 0 \leq t \leq x \leq 1\}$ such that $M_{1}=\max _{x, s \in G}\left|\lambda K_{1}(x, t)\right|<\infty, M_{2}=\max _{x, s \in G}\left|\mu K_{2}(x, t)\right|<\infty$, 
(A3) The function $g: J \rightarrow \mathbb{R}$ is continuous.

Theorem 5..1 Assume that (A1), (A2) and (A3) hold. If

$$
\left(\frac{e^{\varepsilon M_{1} x}-e^{\varepsilon M_{1} a}}{\varepsilon}+\frac{e^{\varepsilon M_{2} \beta}-e^{\varepsilon M_{2} a}}{\varepsilon}\right)<1 .
$$

Then the initial value problem (1)-(2) has a unique solution.

Proof. Let the operator $A: X \longrightarrow X$. To do this, it is evident that $\left(A u^{c}\right)(x) \in \mathbb{R}_{\mathcal{F}}$ for all $x \in J$ and thus $A u^{c}: J \longrightarrow \mathbb{R}_{\mathcal{F}}$ be defined by

$$
\begin{aligned}
& \left(A u^{c}\right)(x)=g^{c}(x)+\lambda \int_{a}^{x} K_{1}(x, s) F_{1}\left(u^{c}(s)\right) d s+\mu \int_{a}^{\beta} K_{2}(x, s) F_{2}\left(u^{c}(s)\right) d s, \\
& D\left(A u_{1}^{c}(x), A u_{2}^{c}(x)\right)=D\left(g^{c}(x)+\lambda \int_{a}^{x} K_{1}(x, s) F_{1}\left(u_{1}^{c}(s)\right) d s+\mu \int_{a}^{\beta} K_{2}(x, s) F_{2}\left(u_{1}^{c}(s)\right) d s, g^{c}(x)\right. \\
& \left.+\lambda \int_{a}^{x} K_{1}(x, s) F_{1}\left(u_{2}^{c}(s)\right) d s+\mu \int_{a}^{\beta} K_{2}(x, s) F_{2}\left(u_{2}^{c}(s)\right) d s\right) \\
& =D\left(\lambda \int_{a}^{x} K_{1}(x, s) F_{1}\left(u_{1}^{c}(s)\right) d s+\mu \int_{a}^{\beta} K_{2}(x, s) F_{2}\left(u_{1}^{c}(s)\right) d s, \lambda \int_{a}^{x} K_{1}(x, s) F_{1}\left(u_{2}^{c}(s)\right) d s\right. \\
& \left.+\mu \int_{a}^{\beta} K_{2}(x, s) F_{2}\left(u_{2}^{c}(s)\right) d s\right) \\
& \leq \int_{a}^{x} D\left(\lambda K_{1}(x, s) F_{1}\left(u_{1}^{c}(s)\right), \lambda K_{1}(x, s) F_{1}\left(u_{2}^{c}(s)\right)\right) d s \\
& +\int_{a}^{\beta} D\left(\mu K_{2}(x, s) F_{2}\left(u_{1}^{c}(s)\right), \mu K_{2}(x, s) F_{2}\left(u_{2}^{c}(s)\right)\right) d s \\
& \leq \int_{a}^{x} M_{1} D\left(u_{1}^{c}(s), u_{2}^{c}(s)\right) d s+\int_{a}^{\beta} M_{2} D\left(u_{1}^{c}(s), u_{2}^{c}(s)\right) d s \\
& =\int_{a}^{x} M_{1} e^{\varepsilon M_{1} s} e^{-\varepsilon M_{1} s} D\left(u_{1}^{c}(s), u_{2}^{c}(s)\right) d s+\int_{a}^{\beta} M_{2} e^{\varepsilon M_{2} s} e^{-\varepsilon M_{2} s} D\left(u_{1}^{c}(s), u_{2}^{c}(s)\right) d s \\
& \leq \int_{a}^{x} M_{1} e^{\varepsilon M_{1} s} D_{\varepsilon}\left(u_{1}^{c}, u_{2}^{c}\right) d s+\int_{a}^{\beta} M_{2} e^{\varepsilon M_{2} s} D_{\varepsilon}\left(u_{1}^{c}, u_{2}^{c}\right) d s \\
& \leq \frac{e^{\varepsilon M_{1} x}-e^{\varepsilon M_{1} a}}{\varepsilon} D_{\varepsilon}\left(u_{1}^{c}, u_{2}^{c}\right)+\frac{e^{M_{2} \beta}-e^{\varepsilon M_{2} a}}{\varepsilon} D_{\varepsilon}\left(u_{1}^{c}, u_{2}^{c}\right) \\
& =\left(\frac{e^{\varepsilon M_{1} x}-e^{\varepsilon M_{1} a}}{\varepsilon}+\frac{e^{\varepsilon M_{2} \beta}-e^{\varepsilon M_{2} a}}{\varepsilon}\right) D_{\varepsilon}\left(u_{1}^{c}, u_{2}^{c}\right) \text {. }
\end{aligned}
$$

Since $\left(\frac{e^{\varepsilon M_{1} x}-e^{\varepsilon M_{1} a}}{\varepsilon}+\frac{e^{\varepsilon M_{2} \beta}-e^{\varepsilon M_{2} a}}{\varepsilon}\right)<1$, the operator $A$ is a contraction mapping. By the Banach fixed point theorem we conclude that the initial value problem (1)-(2) has a unique solution. Similarly, we can proof the procedure of $u^{d}(x, t)$. Now, we will discuss the convergence of HAM for Eq.(1) in the fuzzy case which divides into two crisp integro-differential equations as Eqs.(8)-(9).

Theorem 5..2 Let the series $\sum_{m=0}^{\infty} u_{m}^{c}(x, t)$ converge to $u^{c}(x, t)$, where $u_{m}^{c}(x, t)$ is produced by the $m$-order deformation (17), and besides $\sum_{m=0}^{\infty} u_{m}^{c}(x, t)$ converges, then $u^{c}(x, t)$ is the exact solution of Volterra-Fredholm integrodifferential equation (8) when using HAM.

Proof. We assume $\sum_{m=0}^{\infty} u_{m}^{c}(x, t)$ converge uniformly to $u^{c}(x, t)$ then

$$
\lim _{m \rightarrow \infty} u_{m}^{c}(x, t)=0, \quad \forall 0 \leq x \leq \beta, 0 \leq t \leq 1
$$


We can write,

$$
\begin{aligned}
\sum_{m=1}^{n} \Psi\left[u_{m}^{c}(x, t)-\chi_{m} u^{c}{ }_{m-1}(x, t)\right]= & \Psi u^{c}{ }_{1}(x, t)+\left(\Psi u^{c}{ }_{2}(x, t)-\Psi u_{1}^{c}{ }_{1}(x, t)\right) \\
& +\left(\Psi u^{c}{ }_{3}(x, t)-\Psi u_{2}^{c}(x, t)\right)+\ldots \\
& +\left(\Psi u^{c}{ }_{n}(x, t)-\Psi u_{n-1}^{c}(x, t)\right) \\
= & \Psi u_{n}^{c}{ }_{n}(x, t) .
\end{aligned}
$$

Hence, from Eq.(21)

$$
\lim _{n \rightarrow \infty} u_{n}^{c}(x)=0
$$

So, using Eq.(22), we have

$$
\sum_{m=1}^{\infty} \Psi\left[u_{m}^{c}(x, t)-\chi_{m} u_{m-1}^{c}(x, t)\right]=\sum_{m=1}^{\infty}\left[u_{m}^{c}(x, t)-\chi_{m} \Psi u_{m-1}^{c}(x, t)\right]=0 .
$$

Therefore from Eq.(22), we can obtain that

$$
\sum_{m=1}^{\infty} \Psi\left[u_{m}^{c}(x, t)-\chi_{m} u_{m-1}^{c}(x, t)\right]=\hbar \sum_{m=1}^{\infty} \Re_{m-1}\left(\overrightarrow{u_{m-1}^{c}}(x, t)\right)=0 .
$$

Since $\hbar \neq 0$ and we have

$$
\sum_{m=1}^{\infty} \Re_{m-1}\left(\overrightarrow{u_{m-1}^{c}}(x, t)\right)=0
$$

By substituting $\Re_{m-1}\left(\overrightarrow{u_{m-1}^{c}}\right)$ into the relation (23) and simplifying it, we have

$$
\begin{aligned}
\Re_{m-1}\left(\overrightarrow{u_{m-1}^{c}}(x, t)\right)= & \sum_{m=1}^{\infty}\left[\frac{\partial u_{m-1}^{c}(x, t)}{\partial x}-\lambda \int_{a}^{x} k_{1}(x, s) F_{1}\left(u_{m-1}^{c}(s, t)\right) d s\right. \\
& \left.-\mu \int_{a}^{\beta} k_{2}(x, s) F_{2}\left(u_{m-1}^{c}(s, t)\right) d s-\left(1-\chi_{m}\right) f^{c}(x, t)\right], \\
= & \left(\sum_{m=1}^{\infty} \frac{\partial u_{m-1}^{c}(x, t)}{\partial x}-\lambda \int_{a}^{x} k_{1}(x, s)\left[\sum_{m=1}^{\infty} F_{1}\left(u_{m-1}^{c}(s, t)\right)\right] d s\right. \\
& -\mu \int_{a}^{\beta} k_{2}(x, s)\left[\sum_{m=1}^{\infty} F_{2}\left(u_{m-1}^{c}(s, t)\right)\right] d s-\sum_{m=1}^{\infty}\left(1-\chi_{m}\right) f^{c}(x, t), \\
= & \frac{\partial u^{c}(x, t)}{\partial x}-\lambda \int_{a}^{x} k_{1}(x, s) F_{1}\left(u^{c}(s, t)\right) d s \\
& -\mu \int_{a}^{\beta} k_{2}(x, t) F_{2}\left(u^{c}(s, t)\right) d s-f^{c}(x, t) .
\end{aligned}
$$

From Eq.(23) and Eq.(24), we have

$$
\frac{\partial u^{c}(x, t)}{\partial x}=f^{c}(x, t)+\lambda \int_{a}^{x} k_{1}(x, s) F_{1}\left(u^{c}(s, t)\right) d s+\mu \int_{a}^{\beta} k_{2}(x, s) F_{2}\left(u^{c}(s, t)\right) d s,
$$

therefore, $u^{c}(x, t)$ must be the exact solution of Eq.(8). Similarly, we can proof the procedure of $u^{d}(x, t)$. Then, $u(x, t)$ must be the exact solution of Eq.(1), and the proof is complete.

\section{ILLUSTRATIVE EXAMPLE} differential equation.

In this section, we present the analytical technique based on HAM to solve fuzzy Volterra-Fredholm integro- 


\section{Example 1.}

Let us consider fuzzy Volterra-Fredholm integro-differential equation:

$$
\frac{\partial \tilde{u}(x, t)}{\partial x}+\tilde{u}(x, t)=\tilde{f}(x, t)+\lambda \int_{0}^{x} x s^{2} \tilde{u}(s, t) d s+\mu \int_{0}^{1} x s \tilde{u}(s, t) d s,
$$

where

$$
\begin{gathered}
\tilde{u}(x, t)=(\underline{u}(x, t), \bar{u}(x, t)), \quad \tilde{f}(x, t)=(\underline{f}(x, t), \bar{f}(x, t)) \\
\underline{f}(x, t)=t\left(\frac{2 x}{3}+1\right), \quad \bar{f}(x, t)=\frac{2 t x}{3}-4 x-t+8, \quad \lambda=0, \quad \mu=1 .
\end{gathered}
$$

with initial conditions

$$
\underline{u}(0, t)=0, \quad \bar{u}(0, t)=8 .
$$

Exact solution of this fuzzy Volterra-Fredholm integro-differential equation is given by

From Eq.(7), we have

$$
\tilde{u}(x, t)=(x t, 8-x t) .
$$

$$
f^{c}(x, t)=\frac{f(x, t)+\bar{f}(x, t)}{2}=4-2 x, \quad f^{d}(x, t)=\frac{\bar{f}(x, t)-\underline{f}(x, t)}{2}=4-t-2 x-\frac{2 t x}{3} .
$$

The exact solution of related crisp equations are as follows

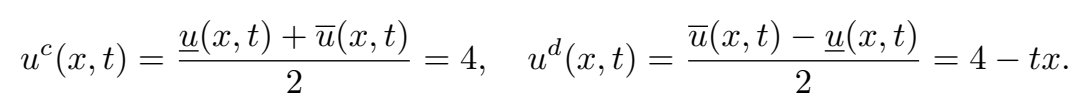

From Eqs.(16) and(25) can be written

$$
N[\varphi(x, t ; q)]=\frac{\partial[\varphi(x, t ; q)]}{\partial x}+\varphi(x, t ; q)-4+2 x-\int_{0}^{1} k_{2}(x, s) F_{2}(\varphi(s, t ; q)) d s,
$$

Now, using $m^{\text {th }}$-order deformation equation and initial conditions, we recursively obtain

$$
\begin{aligned}
& u_{0}^{c}(x, t)=u^{c}(0, t)=\frac{u}{(0, t)+\bar{u}(0, t)} \\
& 2 \\
& u_{1}^{c}(x, t)=0, \\
& u_{2}^{c}(x, t)=0,
\end{aligned}
$$

Thus the approximate HAM solution

$$
Y_{m}^{c}(x, t)=\sum_{n=0}^{m} u_{n}^{c}(x, t)=4
$$

The approximate solution same as exact solution. Similarly, to approximate $u^{d}(t, r)$,

$$
N[\varphi(x, t ; q)]=\frac{\partial[\varphi(x, t ; q)]}{\partial x}+\varphi(x, t ; q)-4+t+2 x+\frac{2 t x}{3}-\int_{0}^{1} k_{2}(x, s) F_{2}(\varphi(s, t ; q)) d s,
$$

Now, using $m^{t h}$-order deformation equation and initial conditions, we recursively obtain

$$
\begin{aligned}
u_{0}^{d}(x, t) & =u^{d}(0, t)=\frac{\bar{u}(0, t)-\underline{u}(0, t)}{2}=4, \\
u_{1}^{d}(x, t) & =\frac{\hbar}{3} x t(3+x), \\
u_{2}^{d}(x, t) & =\frac{\hbar}{72} x t\left(\hbar\left(72+45 x+8 x^{2}\right)+72+24 x\right),
\end{aligned}
$$


Thus the approximate HAM solution when $\hbar=-1$

$$
Y_{m}^{d}(x, t)=\sum_{n=0}^{m} u_{n}^{d}(x, t)=u_{0}^{d}(x, t)+u_{1}^{d}(x, t)+\cdots+u_{m}^{d}(x, t) \approx 4-t x .
$$

Note that, we can control the convergence region of HAM series solution by the auxiliary parameter $\hbar$.

\section{CONCLUSION}

Homotopy analysis method has been performed to find approximate analytical solutions for fuzzy VolterraFredholm integro-differential equations. The reliability of the method and reduction in the size of the computational work give this method a wider applicability. The method is very powerful and efficient in finding analytical as well as numerical solutions for wide classes of linear and nonlinear fuzzy integro-differential equations. Obtained results show that we can control the convergence region of HAM series solution by the auxiliary parameter $\hbar$. The illustrative example and convergence theorem show the efficiency and accuracy of the HAM.

\section{REFERENCES}

[1] S. Chang and L. Zadeh, "On fuzzy mapping and control," IEEE Trans. Systems, Man cybernet, (1972), 2, pp. 30-34.

[2] D. Dubois and H. Prade, "Operations on fuzzy numbers," Int. J. systems Science, (1978), 9, pp. $613-626$.

[3] D. Dubois and H. Prade, "Towards fuzzy differential calculus. Integration of fuzzy mappings," Fuzzy Sets and Systems, (1982), 8, pp. 1-17.

[4] O. Kaleva, "Fuzzy differential equation," Fuzzy Sets and Systems, (1987), 24, pp. 301-317.

[5] S. Narayanamoorthy and S. Sathiyapriya, "A pertinent approach to solve nonlinear fuzzy integro-differential equations," Springer Plus. (2016), 5, pp. 1-17.

[6] T. Allahviranloo, S. Abbasbandy and S. Hashemzehi, "Approximating the solution of the linear and nonlinear fuzzy Volterra integro-differential equations using expansion method," Abstr. Appl. Anal. (2014), pp. 1-8.

[7] M. Das and D. Talukdar, "Method for solving fuzzy integro-differential equations by using fuzzy Laplace transformation," Int. J. Sci. Tech. (2014), 5, pp. 291-295.

[8] E. Hussain and A. Ali, "Homotopy analysis method for solving fuzzy integro-differential equations," Modern Appl. Sci. (2013), 7, pp. 15-25.

[9] S. Behiry and S. Mohamed, "Solving high-order nonlinear Volterra-Fredholm integro-differential equations by differential transform method," Nat. Sci. (2012), 8, pp. 581-587.

[10] H. Rahimi, M. Khezerloo and S. Khezerloo, "Approximating the fuzzy solution of the non-linear fuzzy Volterra integro-differential equation using fixed point theorems," Int J Indus Math. (2011), 3(3), pp. 227-236.

[11] M. Hashemi and S. Abbasbandy, "The solution of fuzzy Volterra-Fredholm integro-differential equations using variational iteration method," Int. J. Math. Comput. (2011), 11, pp. 29-38.

[12] A. Hamoud and K. Ghadle, "modified Adomian decomposition method for solving fuzzy Volterra-Fredholm integral equations," Journal of the Indian Math. Soc. (2018), 85(1-2), pp. 01-17.

[13] A. Hamoud and K. Ghadle, "Existence and uniqueness of solutions for fractional mixed Volterra-Fredholm integro-differential equations," Indian J. Math. (2018), 60(3), pp. 375-395.

[14] A. Hamoud, K. Ghadle, M. Bani Issa and Giniswamy, "Existence and uniqueness theorems for fractional Volterra-Fredholm integro-differential equations," Int. J. Appl. Math. (2018), 31(3), pp. 333-348.

[15] A. Hamoud, K. Ghadle and S. Atshan, "The approximate solutions of fractional integro-differential equations by using modified Adomian decomposition method," Khayyam J. Math. (2019), 5(1), pp. 21-39.

[16] A. Hamoud and K. Ghadle, "Existence and uniqueness of the solution for Volterra-Fredholm integro-differential equations," Journal of Siberian Federal University. Mathematics \& Physics, (2018), 11(6), pp. 692-701.

[17] A. Hamoud and K. Ghadle, "Some new existence, uniqueness and convergence results for fractional VolterraFredholm integro-differential equations," J. Appl. Comput. Mech. (2019), 5(1), pp. 58-69.

[18] A. Hamoud, M. Bani Issa and K. Ghadle, "Existence and uniqueness results for nonlinear Volterra-Fredholm integro-differential equations," Nonlinear Functional Analysis and Applications, (2018), 23(4), pp. 797-805.

[19] A. Hamoud and K. Ghadle, "Usage of the homotopy analysis method for solving fractional Volterra-Fredholm integro-differential equation of the second kind," Tamkang Journal of Mathematics, (2018), 49(4), pp. 301-315.

[20] A. Hamoud and K. Ghadle, "Recent advances on reliable methods for solving Volterra-Fredholm integral and integro-differential equations," Asian J. Math. Comput. Res. (2018), 24, pp. 128-157.

[21] Y. Chalco-Cano and H. Roman-Flores, "On new solutions of fuzzy differential equations," Chaos, Solitons and Fractals, (2008), 38, pp. 112-119. 
[22] J. Liao, ”Beyond Perturbation: Introduction to the Homotopy Analysis Method," CRC Press, Boca Raton: Chapman Hall, 2003.

[23] K. Ghadle and A. Hamoud, "Study of the approximate solution of fuzzy Volterra-Fredholm integral equations by using (ADM)," Elixir Appl. Math. (2016), 98, pp. 42567-42573.

[24] A. Kilbas, H. Srivastava and J. Trujillo, ”Theory and Applications of Fractional Differential Equations," NorthHolland Math. Stud. 2042006.

[25] S. Nanda, "On integration of fuzzy mappings, Fuzzy Sets and Systems," (1989), 32, pp. 95-101.

[26] Y. Shao and H. Zhang, "Fuzzy integral equations and strong fuzzy hen-stock integrals," Abstr. Appl. Anal. (2014), pp. 1-8.

[27] L. Zadeh, "The concept of a linguistic variable and its application to approximate reasoning," Inform. Sci., (1975), 8, pp. 199-249.

[28] Y Zhou, "Basic Theory of Fractional Differential Equations," Singapore: World Scientific, 6, 2014. 